\title{
DESCRIBING CRYPTOBIOSIS AS A TIME BASED PROTECTION SYSTEM USING PETRI NETS
}

\author{
Bengt Carlsson ${ }^{\mathrm{a}}$, K. Ingemar Jönsson ${ }^{\mathrm{b}}$ and Keith Clark ${ }^{\mathrm{c}}$ \\ ${ }^{a}$ School of Engineering Blekinge Institute of Technology Soft Center S-37225 Ronneby, Sweden bca@bth.se \\ ${ }^{b}$ Department of Mathematics and Science, Kristianstad University,S-29188 Kristianstad, Sweden.ingemar.jonsson@hkr.se \\ ${ }^{c}$ Department of Computing, Imperial College, London klc@doc.ic.ac.uk
}

Keywords: Cryptobiosis, Petri net, Time based security model.

\begin{abstract}
Cryptobiosis represents the state of a living organism when it shows no visible signs of metabolic life, but maintains a capacity to return to an active, metabolic state. This peculiar state, although known from a wide variety of organisms, has received little attention from a theoretically biological perspective. A description based on a Petri net setting and a time based security model is proposed. In order to protect against a prolonged exposure time, the pathways for chemical reactions involved must fulfil their actions during a limited detection and response time to fulfil the protected state of entering/leaving cryptobiosis.
\end{abstract}

\section{INTRODUCTION}

Within biology the term cryptobiosis, or hidden life, represents the state of an organism when it shows no metabolic signs of life, while still maintaining a capacity to return to normal metabolic activity (Keilin 1959, Clegg 2001). Organisms with a capacity to enter cryptobiosis are found in a variety of prokaryote, plant, and animal taxa, and has evolved as an adaptation to survive under temporally hostile conditions, e.g., in very dry or cold environments (Alpert 2005). One of the most common environmental agents inducing cryptobiosis is desiccation, leading to a variant of cryptobiosis called anhydrobiosis. The cryptobiotic state is characterised by temporally arrested metabolism, growth, reproduction and senescence (Keilin 1959, Crowe 1971). The lack of metabolism during cryptobiosis can be described as temporary "death" representing a unique biological state between life and death, a potentially reversible death (Neuman 2006).

The biochemical and physiological mechanisms allowing cryptobiotic organisms to survive in an ametabolic and (in the case of anhydrobiosis) more or less completely dry state remain poorly understood (Schill et al. 2004, Watanabe et. al. 2002, Guppy 2004). Also, the problems connected with extreme desiccation and a complete shut-down of the metabolic machinery have rarely been analysed theoretically. However, some criteria are necessary to fulfil for successful cryptobiosis to take place. For instance, the organism must either prevent cellular damage at the entrance of cryptobiosis and during the cryptobiotic state, or be able to repair the damage that is potentially expressed when reactivated. It must also be able to respond to one or more triggering signals connected with the cryptobiotic period. We will here describe a possible approach to a protection system during an induction phase, a dormancy phase and a reactivating phase of cryptobiosis.

In order to return to an active life, an organism in cryptobiosis needs to interpret signals from the environment including reactivating itself from the ametabolic state, i.e. recovering original biological functions despite the lack of basic metabolic machinery. The organism must be "raised from the dead", by responding to an environmental signal announcing favourable life conditions, e.g. a drop of water for a dehydrated organism. It has been suggested that cryptobiosis entails reversible computation with a bootstrapping involving a recursive hierarchy (Neuman 2006). This is similar to models within computer science including creating more complex tools from simple tools (bootstrapping), sub and superclasses within object oriented programming (hierarchies), and logic programming using recursive functions. We will here use a Petri net as a tool for describing the 
reversible processes of cryptobiosis, without going into the details of the metabolic pathways involved.

Biological systems modelled by Petri nets were introduced by Reddy et al (1993). By using Petri net it was possible to dynamically model the intrinsic behaviour of e.g. metabolic pathways within a cell. In Heiner et al (2004) the metabolic pathways conducting an apoptosis (genetically programmed cell death) is modelled using a qualitative Petri net.

In section 2 the different phases of cryptobiosis are outlined followed by a description of a time based protection system. Next, a Petri net is introduced for modelling metabolic protection and finally a discussion and concluding part sum up the proposed description.

\section{CRYPTOBIOSIS}

Cryptobiosis may be induced by several environmental factors e.g. low temperature (cryobiosis), lack of oxygen (anoxybiosis) or lack of water (anhydrobiosis). All of these factors may force the organism to an arrested metabolism. Here, we will use anhydrobiosis as an example to describe cryptobiosis within a Petri net setting using a time based security model.

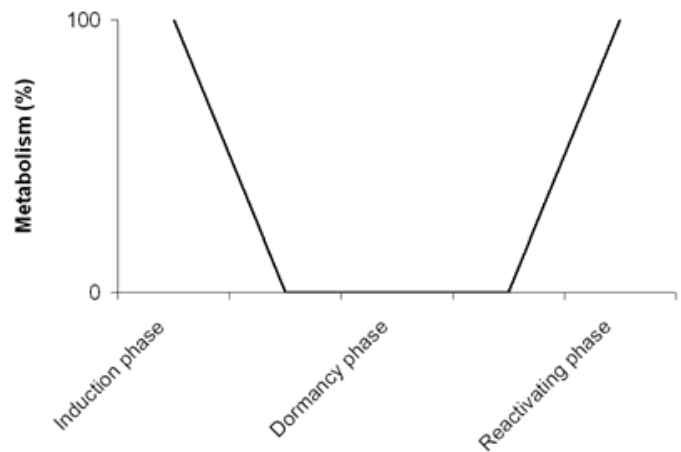

Figure 1. An idealized figure of changes in metabolism during the induction, dormancy, and reactivating phases of cryptobiosis. A more realistic curve would likely involve non-linear patterns of metabolic changes during induction and reactivation.

Figure 1 shows the three general phases connected with cryptobiosis, where the induction phase prepares the organism for an ametabolic state as a result of e.g. desiccation. Note that the factor inducing changes in metabolic rate, e.g. reduced hydration level, is not shown in Figure 1. During the dormancy phase the organism is inactive due to a lack of metabolic activity, but continued desiccation may occur because metabolism will stop well before the animal is completely dehydrated. The reactivating phase involves both the reverse processes of the induction phase and a repair process depending on damages arisen during the induction and dormant phases.

The pattern of water loss at the induction of cryptobiosis as a result of dehydration (anhydrobiosis) has been well documented (Wright 1989, Wharton 1996). Initially the evaporation of water is high, but at some point the organism has mobilised a first protection mechanism (mainly based on morphological changes) which dramatically reduces the rate of further dehydration. Wright (1989) termed this point the "permeability slump". After this point, the rate of dehydration is much reduced. The remaining time, until the organism has lost so much of its water that metabolism is arrested, is decisive of whether the organism will enter a cryptobiotic state or die. During this time, the organism must mobilise the mechanisms that should protect it as it approaches the dry anhydrobiotic state. Figure 2 describes the temporal change in hydration level of an anhydrobiotic organism exposed to a desiccating agent.

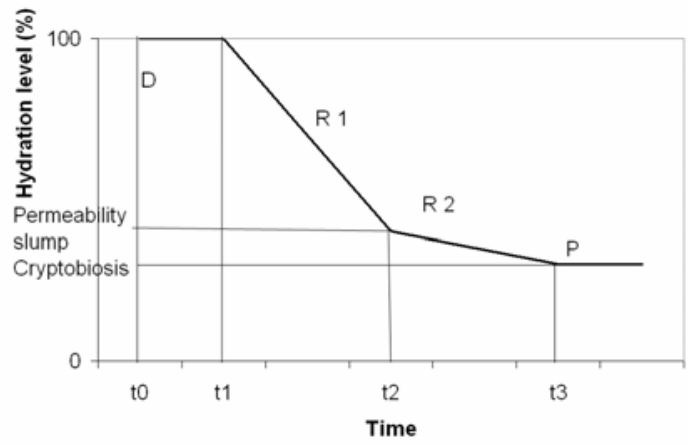

Figure 2. Patterns of water loss over time in an anhydrobiotic organism exposed to a desiccating agent, with phases of detection (D) and responses $\left(\mathrm{R}_{\mathrm{i}}\right)$, creating a state of cryptobiotic protection $(\mathrm{P})$.

In both computer science and in cryptobiotic organisms, a detection mechanism must recognize the attack or stress input, after which different response mechanisms must be activated (R1 and R2 in Figure 2). In cryptobiotic organisms these preventing mechanisms together form the protection system which must be activated before the metabolism disappears. 


\section{A TIME BASED PROTECTION SYSTEM}

To describe, in general terms, a cryptobiotic protection system, a time based security model (Schwartau 1999) may be used, where a protection mechanism is successful only as long as the sum of the time needed by the detection mechanism and the response mechanism does not exceed the time limit of the protection.

In this model the amount of exposure time E, which may be positive or negative, determines the outcome of an external attack. The exposure time defines a time period under which the organism is exposed to the environmental stress without a mobilized protection system. The exposure time is determined by three factors; a protection system P, a detection mechanism $\mathrm{D}$, and a response mechanism $\mathrm{R}$, see Figure 2. The purpose of a protection system, independent of being within computer science or life science, is to protect involved assets. This is valid for a certain amount of time, i.e. the involved assets lose their values or an organism loses the opportunity to enter a cryptobiotic state. If detection and response time is short enough, depending on the current attack strength, the protection system will not be invaded or exposed. So a negative exposure time means that the protection mechanism manages to keep the system secured during the time when detection and responses are established.

Let $\Delta P$ denote the duration of protection of a system caused by an attack with strength $a$ starting at time $t_{0}$ and with protection strength $s$. Let $\Delta D$ denote the time it takes to detect an attack with strength $a$ under given circumstances. Finally, let $\Delta R$ denote the time it takes to implement sufficient measures to eliminate the negative consequences of the attack with strength $a$ after the signal has been detected. Then

$\Delta E\left(a, s, t_{0}, t\right)=\Delta D\left(a, s, t_{0}, t\right)+\Delta R\left(a, s, t_{0}, t\right)-\Delta P\left(a, s, t_{0}, t\right)$

Equation (1) captures exposure time $\Delta E\left(a, s, t_{0}, t\right)$ as a relation between the three mechanisms above and the time intervals $t_{0}$ and $t$ where $t>t_{0}$. The system will be safe if and only if $\Delta E<0$, i.e., if the system is not exposed to an attack without a protection system.

As already pointed out in Figure 2, a protection system consisting of detection, responses and protection are outlined for the induction phase. During the first period of time $\left(t_{0}\right.$ to $\left.t_{1}\right)$ the organism has not yet started to lose its internal water, but a detection mechanism (D) recognizes the presence of a stress signal. This may be, e.g., the evaporation of surrounding water. In the next interval $\left(t_{1}\right.$ to $\left.t_{2}\right)$, the organism starts to lose its body water and a first response mechanism $\left(R_{1}\right)$ is mobilised. When the establishment of this mechanism is completed (at $t_{2}$ ), the rate of dehydration is much reduced, but continues until the organism has reached a level of hydration at which metabolism stops. Since metabolism is arrested well before the organism is completely dry (Clegg 1986), the loss of water may continue also after the cryptobiotic state has been reached. In the interval between $t_{2}$ and $t_{3}$ the second response $\left(R_{2}\right)$, representing the biochemical preparations for the dry cryptobiotic state, takes place. If these preparations are successful, i.e., if the second protection system has been established properly, the organism enters cryptobiosis. The time available for the necessary actions (detection + responses) will be determined by the rate of desiccation from $t_{0}$ to $t_{3}$, influenced in part by the strength of the desiccation agent, and in part by the ability of the organism to reduce the effect of this agent. If detection or response is delayed the protection system will not be ready, and the organism will die or be damaged before reaching a state of cryptobiosis. Equation (2) formalizes this organism system:

$\Delta E\left(a, s, t_{0}, t_{3}\right)=\Delta Q\left(a, s, t_{0}, t_{1}\right)+\Delta R_{1}\left(a, s, t_{1}, t_{2}\right)+\Delta R_{2}\left(a, s, t_{2}, t_{3}\right)-\Delta P\left(a, s, t_{0}, t_{3}\right)$

The organism will enter a state of cryptobiosis if $\Delta E$ is negative, i.e. if the time needed to establish the protection mechanism $\Delta P$ exceeds the sum of the time for the detection $\Delta D$ and response mechanism $\Delta R_{i}$. The main difference in the interpretation of Eq. (1) (representing a computer science model) and Eq. (2) (representing a biological model) is that in the former model the protection system is present already at the beginning, with an expected time of function. In the biological model, the protection system is not described as present from the start, but is established only after the stress agent is detected, with an expected time of establishment $(\Delta P)$. However, from the perspective of $P$, the responses $R_{1}$ leads to an intermediate protection system (activated at the "permeability slump") that conceptually resembles that of computer systems, since its location in Figure 2 defines a remaining time within which the $R_{2}$ responses must take place.

Processes during the reactivating part must include both activating the reversible processes of the induction phase, and repairing of damage caused by positive exposure time during induction and dormancy phases. 


\section{USING A PETRI NET FOR MODELLING METABOLIC PROTECTION}

A Petri net consists of nodes and arcs where nodes are of two types; places $p_{i}$ and transitions $t_{i}$. Tokens represent activity of the sequence of transitions. Places, represented by circles, are passive system elements while transitions represented by boxes are active system elements, e.g. in a metabolic setting chemical compounds and chemical reactions. Arcs connect nodes of different type. The activity of a Petri net is a flow of tokens over the pathways of the net

To start with, the organism must detect the state of stress, or cryptobiosis stimuli, which in the case of anhydrobiosis is represented by the initiation of desiccation (loss of water), and the intensity of the stress factor. If the humidity surrounding the organism is very low the process towards a dry and cryptobiotic state will be more rapid, and the organism then has less time to prepare itself before metabolism ceases. Preparations for the dry state therefore often involve morphological changes that reduce the rate of water loss (e.g., Wright 2001). Previous research has shown that this is a critical phase, where the cell must be able to mobilize the necessary metabolic changes that allow it to maintain structural integrity and enter a dry state without lethal damage. These changes, or set of places $p_{i}$ and transitions $t_{j}$ in a Petri net setting, are represented as different pathways in Figure 3.

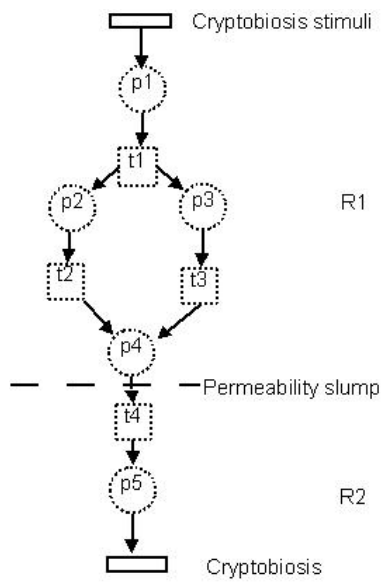

Figure 3 Induction phases of cryptobiosis. The dotted circles and squares indicate examples of metabolic pathways.
The dynamics of a Petri net allows transitions to split between multiple places or places requires more than one transition to enter a state. The number of places and transitions are not known nor are the structures of the pathways. When two arcs arrive at a place it is a synchronisation step - only when the tokens from the converging pathways have both arrived at the place, does the transition on the outgoing arc fire.

Examples of pathways are molecules (e.g., sugars, Crowe 2002) that replace structural water in cell membranes, and molecular chaperones (stress proteins) that prevent aggregation of proteins (Goyal et al. 2005). All the details involved are outside the scope of this work, but several pathways that slow down or disappear during transforming from R1 to R2 are involved.

Thus, the protection system allowing cryptobiotic survival includes several protection parameters, the induction of which relies on metabolic activity. Note that this has to be done before entering the cryptobiotic state, i.e. the protection system must act proactively.

Compared to the induction phase and the reactivating phase, which normally amounts to minutes or hours, the dormancy phase may be much more extended in time and may last for weeks, months or years (Guidetti and Jönsson 2002, Watanabe 2006).

The reactivation from the anhydrobiotic state also provides a challenge for the organism, the physiological details of which has yet to be described. At some point in time the metabolism must restart as a reaction to changed conditions, e.g., to rehydration. In principle this is the reverse function of the induction phase restarting the protection system. All the necessary biochemical components for starting up the system are present, and some of the protection components may be used for energy production or for repairing DNA and other parts of the cell.

In principle the protection system needs to be restored to its pre-conditions with one major addition, damaged parts or processes must be repaired. This is part of the existing pathways or added as new pathways enlarging the Petri net. The protection system will not be restored without successful repair facilities and, in order to have a functioning repair mechanism, some restored protection mechanisms must be present. Meanwhile there should be a replacement process where water is tied to the organism again. Finally, when the metabolism is restored to 100 percent, we are back to a fully active organism. 


\section{DISCUSSION AND CONCLUSION}

As pointed out by Neuman (2004), cryptobiosis involves reversible computation with a suggested bootstrapping involving a recursive hierarchy. As a model for analysing the behaviour of cryptobiotic systems, we propose using a Petri net, initiated by one or more input signals, computing a number of pathways for the induction and reactivating phases.

In computer science bootstrapping refers to a process where an initial system activates a more constant system maintaining fundamental skill. The initial process may be simple, activating a complex system step by step. Activating metabolism in a cryptobiotic organism may cause a chain reaction that step by step brings back the organism to its initial conditions. To model cryptobiosis both a Petri net setting and a time based security model is used, i.e. the chemical reactions involved and time for accomplishing the involved tasks.

The input stress to which cryptobiotic organisms are exposed could be seen as an "attack" against a system. The organism must first detect the attack and then respond to it in order to protect the system. Organisms with a fast enough detection and response will fulfil the protection mechanism's conditions. In a dynamic environment, where varying environmental conditions are presupposed, the robustness of the protection system may be modelled. This robustness is dependent of the strength of stress factor, possible threshold functions connected Petri net pathways, detection time and the success of all responses during a limited amount of time.

Currently the biological phenomenon of cryptobiosis lacks comprehensive models for describing involved processes, both at a general level and within more specific system. Such models may be found within computer science. Petri net settings may describe the different biological aspects and processes connected with cryptobiosis, and allow simulation of them. By putting more realism into the models, a future progress of both models and simulation tools may result in a better understanding of resource control within protection systems, an important issue both within life science as within computer science.

\section{REFERENCES}

Alpert, P., 2005, The limits and frontiers of desiccationtolerant life. Integr. Comp. Biol. 45: 685-695.

Clegg, J.S., 1986, The physical properties and metabolic status of Artemia cysts at low water contents: the "
Water Replacement Hypothesis". In: LEOPOLD, A. C. (ed.) Membranes, Metabolism, and Dry Organisms, pp. 169-187. Comstock Publ. Ass., London.

Clegg, J.S., 2001, Cryptobiosis - a peculiar state of biological organization, Comp. Biochem. and Physiol. Part.B. vol 128 (4).

Crowe, J.H., 1971, Anhydrobiosis: an unsolved problem. Am. Nat. 105: 563-573.

Crowe L.M., 2002, Lessons from nature: the role of sugars in anhydrobiosis. Comp. Biochem. Physiol. A 131: 505-513.

Goyal, K., Walton, L.J., Browne, J.A., Burnell, A.M., and Tunnacliffe, A., 2005, Molecular anhydrobiology: identifying molecules implicated in invertebrate anhydrobiosis. Integr. Comp. Biol. 45: 702-709.

Guidetti, R. and Jönsson, K.I.,, 2002, Long-term anhydrobiotic survival in semi-terrestrial micrometazoans. J. Zool. 257: 181-187.

Guppy, M., 2004, The biochemistry of metabolic depression: a history of perceptions, Comp. Biochem. and Physiol. Part.B. vol 139 (3).

Heiner, M., Koch, I. and Will J., 2004, Model validation of biological pathways using Petri nets - demonstrated for apoptosis. Biosystems 75 p. 15-28.

Keilin, D., 1959, The problem of anabiosis or latent life: history and current concept. Proc. R. Soc. Lond. B. 150: 149-191.

Neuman; Y., 2004, Meaning making in the immune system, Perspect. Biol. Med. 47.

Neuman, Y., 2006, Cryptobiosis: A new theoretical perspective, Progress in Biophysics and Molecular Biology 92.

Reddy,V.N., Mavrovouniotis, M.L., Liebman, M.N., 1993, Petri net representation in metabolic pathways., Proceedings of the First International Conference on Intelligent Systems for Molecular Biology, AAAI Press p. 328-336.

Schill, R.O. Steinbruck, G.H.B., and Köhler, H-R, Stress 2004, gene (hsp70) sequences and quantitative expression in Milensium tardigradum (Tardigrade) during active and cryptobiotic stages, Journal of Experimental Biology 207.

Schartau, W., 1999, Time based security. Practical and Provable Methods to Protect Enterprise and Infrastructure, Network and Nation, Interpact Press.

Watanabe, M., 2006, Anhydrobiosis in invertebrates. Appl. Entomol. Zool. 41: 15-31.

Watanabe, M., Kikawada, T., Minagawa, N., Yokuhiro, F., and Okuda, T., 2002 Mechanism allowing an insect to survive complete dehydration and extreme temperatures, J. Exp. Biol. 205: 2799-2802

Wharton, D., 1996, Water loss and morphological changes during desiccation of the anhydrobiotic nematode Ditylenchus dipsaci. J Exp Biol. 199: 1085-93.

Wright, J.C., 1989, Desiccation tolerance and and waterretentive mechanisms in tardigrades. J. exp. Biol. 142: 267-292.

Wright, J.C., 2001, Cryptobiosis 300 years on from van Leuwenhoek: what have we learned about tardigrades? Zool. Anz. 240: 563-582. 